\title{
Chemostratigraphy of Sediments in Benin West -1, Benin Flank: Paleoenvironmental Implications
}

\author{
O. Odedede* \\ Department of Geology, Delta State University, Abraka, Delta State, Nigeria \\ Received 16 July 2015, accepted in final revised form 10 October 2015
}

\begin{abstract}
Chemostratigraphical study was carried out on sediments of Benin west -1 well in order to understand their paleoenvironmental setting and geochemical zonations. Six chemozones (CHM 1, CHM 2, CHM 3, CHM 4, CHM 5 and CHM 6) were recognized in the stratigraphic column. Sedimentological attributes and maxima peaks and / or fluctuating trends of $\mathrm{Zn}, \mathrm{SiO}_{2}, \mathrm{~K}_{2} \mathrm{O}, \mathrm{Sr}$ adjudged CHM 3 zone as a potential lowstand systems tract. Synergy of sedimentological and chemostratigraphical fingerprints suggests a marine to distributary channel environment of deposition.
\end{abstract}

Keywords: Chemozones; Palaeoenviromental; sediments; Sedimentological; Marine; Lowtstand systems tract.

(C) 2016 JSR Publications. ISSN: 2070-0237 (Print); 2070-0245 (Online). All rights reserved.

doi: http://dx.doi.org/10.3329/jsr.v8i2.24236 J. Sci. Res. 8 (2), 159-169 (2016)

\section{Introduction}

Chemostratigraphy involves the application of major and trace elements geochemistry for the characterization and subdivision of sedimentary sequences into geochemically distinct units, and correlation of strata in sedimentary basins [1]. Sedimentary rocks records sensitive, subtle changes in environment of deposition, syn-depositional and post-depositional environment. The characterization of these subtle geochemical heterogeneities enables apparently uniform thick successions to be subdivided and correlated between wells [1].

In recent years, much has been written on the use of elemental geochemistry in sediments and water columns as a proxy for depositional redox conditions [2], provenance [3,4] and shale resource plays [5] among others. Over the past five decades, the Benin Flank has been the subject of numerous studies as a result of its

*orresponding author: odededeo@yahoo.com 
long history of tar sand and hydrocarbon exploration. Coker and coauthor have investigated a link between the stratigraphy of the Benin Basin and its tectonic history during the opening of the Equatorial Atlantic [6]. The host sands for the asphalt impregnated sandstones was regarded as transitional to fully marine in nature [7]. Following a series of academic and industrial studies over the past five decades of exploration, most studies from hydrocarbon wells within the northwestern sector of the basin are fragmentary [8] and there is dearth of information on the paleoenvironmental setting of the sediments. In addition, most of the reservoirs in the Benin Flank are barren/lean in terms of fossils, and also have limited lithological variations. More so, variable physical mechanisms of sediment dispersal due to differing environments of deposition lead to fundamentally unique sets of completely stacked lithofacies according to literature [9]. In the light of the aforementioned reasons, correlations are often difficult based on conventional geoscientific methods. In order to account for this challenges, synergy of sedimentology and chemostratigraphic data was employed to provide a robust understanding of the sediments for effective evaluation of subsurface geology of the studied area (Fig. 1).The aim of the present research is to use geochemical data to construct chemostratigraphic zonations, delineate probable reservoirs and interpret paleoenvironment of deposition.

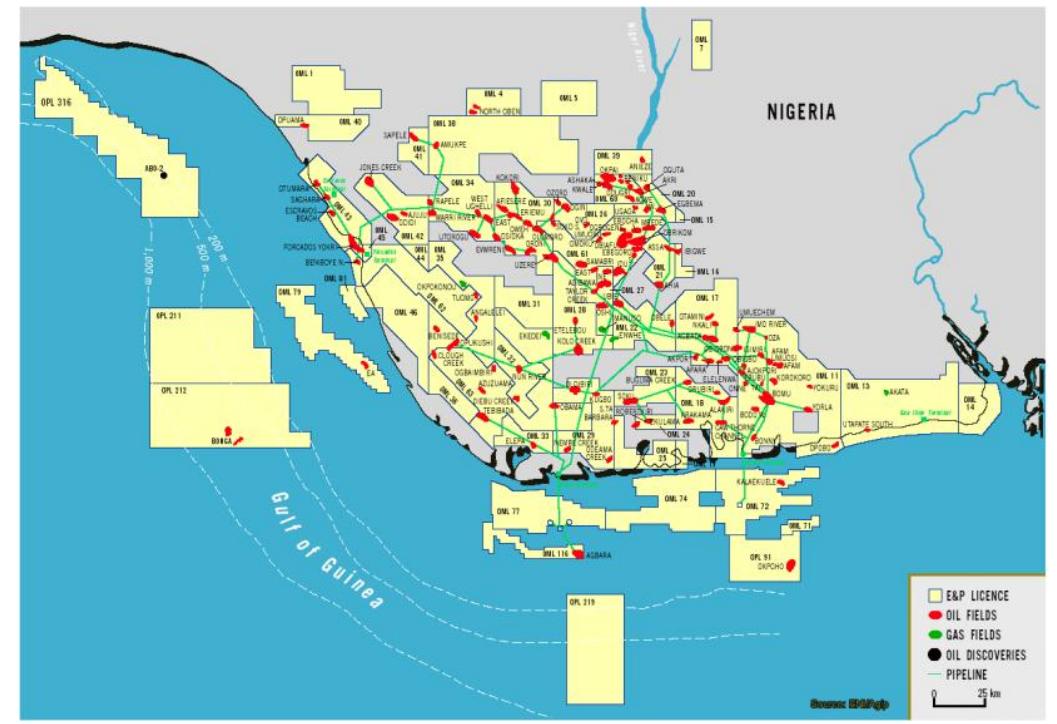

Fig. 1. Map of Niger Delta showing location of Benin West-1.

\section{Geological Setting}

Benin Flank is an east - northeast trending hinge line south of the West Africa basement massif (Fig. 1). The Benin west -1 well lies within the flank of the Anambra Basin. Tectonism in Southern Nigeria probably started in early Cretaceous times with 
the formation of the Benue-Abakaliki Trough. This structure is one arm of a rift-riftrift triple junction associated with the separation of the Africa and South America continent and subsequent opening of the South Atlantic [10,11]. The Cretaceous compressional folding that affected the Lower Benue Trough took place during the Santonian [12] and the main fold structure produced was the NE-SW trending Abakaliki anticlinorium which resulted from inversion of the Abakaliki Trough [12]. The second tectonic episode (renewed rifting) occurred during post-Santonian time and produced the main post-Santonian depocentre, the Anambra Basin on the western flank and a smaller Afikpo Basin on the Southeastern flank of the Abakaliki anticlinorium [13,12]. The Santonian uplift resulted in the erosion of over $2000 \mathrm{~m}$ of sediments from the Abakaliki folded belts which was deposited in the Anambra Basin [12]. Three major sedimentary cycles have been recognized in the basin since early Cretaceous times $[14,10]$. The first cycle was during the Albian time which involved mainly marine deposition [14]. The second cycle began with a Campanian marine transgression and included the growth of a proto-Niger Delta in the northern part of the basin [14]. The third cycle episode marked by Paleocene transgression, deposited the Imo Shale (Fig. 2) with subsurface lateral equivalents of the Akata Formation [14]. A regressive phase during the Eocene resulted in the deposition of Ameki Formation [14]. The Paleogene sediments encountered in the Benin west -1 formed the cornerstone of this study.

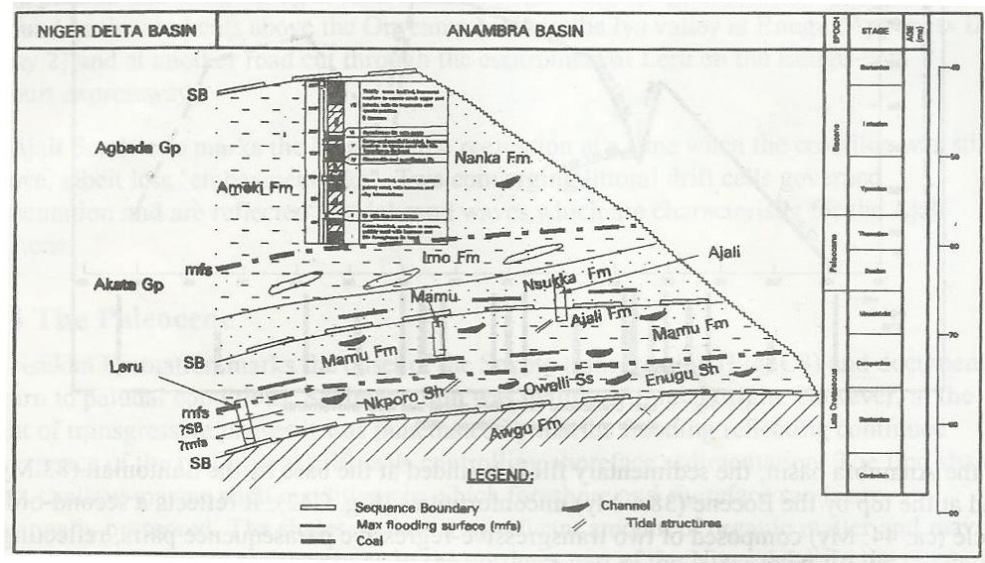

Fig. 2. Sequence stratigraphic synopsis for the Anambra Basin [15].

\section{Experimental}

Thirteen fresh representative samples (sandstones, shaly sandstones and shales) were carefully selected on the basis of sedimentary attributes from the Benin west -1 (Fig. 3 ). A total of 13 samples were analyzed for major element represented as oxides and trace elements (Tables 1-2). Prior to analyses, ditch cutting samples were dried and pulverized. Major and trace element compositions were analyzed at Activation 
Laboratories Ltd., Ancaster, Ontario, Canada using Transducer Inductively Coupled Plasma (TD- ICP) and Fusion Inductively Coupled Plasma (Fusion - ICP) methods. Ten major and thirty six trace elements were determined. The precision is $<5 \%$ for all analyzed elements.

\section{Results and Discussions}

The Benin West-1 well penetrated sedimentary succession from depth of 134 to 2649 $\mathrm{m}$ and the various sedimentary successions encountered (Fig. 3) have been reported elsewhere [16].

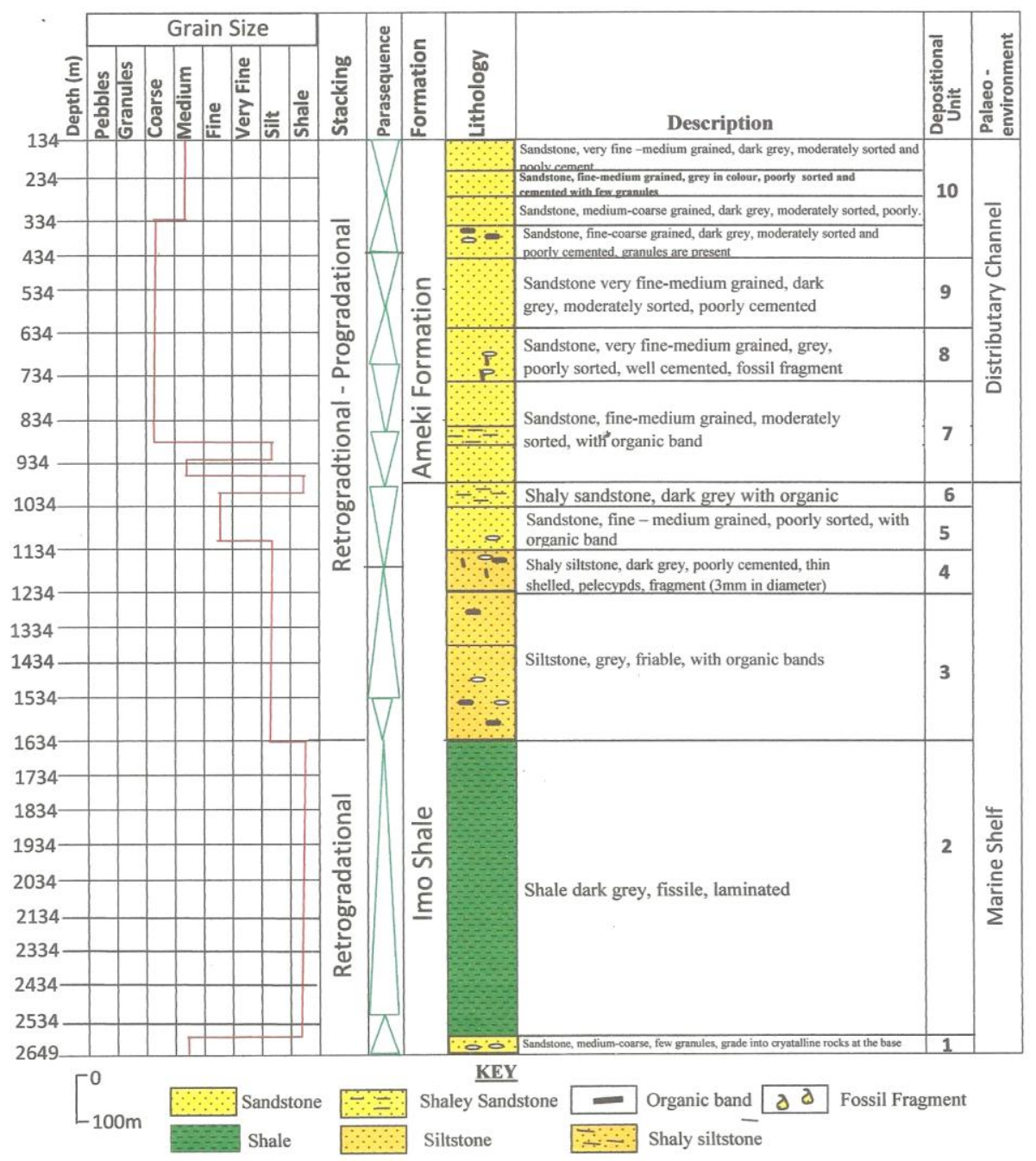

Fig. 3. Stratigraphic Log for Benin West- 1 Well [16]. 


\subsection{Chemostratigraphy of sediments in Benin west-1}

A complete record of geochemical results obtained from the Benin west -1 sediments are presented in Tables 1-2. $\mathrm{SiO}_{2}$ ranges from 27.22 to $74.94 \%$. It increases in the sandy unit (top section) and decreases (Fig. 4) downward particularly in the shaly unit. $\mathrm{Al}_{2} \mathrm{O}_{3}$ ranges from 6.53 to $19.61 \%$. Maximum enrichment was recorded in the shaly unit and fluctuates in the sandy units. This may indicate ferruginous materials and clay cements. $\mathrm{Na}_{2} \mathrm{O}$ concentration ranges from 0.11 to $1.37 \%, \mathrm{Na}_{2} \mathrm{O} / \mathrm{Al}_{2} \mathrm{O}_{3}>0.04 \%$ and indicate presence of plagioclase feldspar [5]. $\mathrm{Fe}_{2} \mathrm{O}_{3}$ ranges from 2.3 to $8.99 \%$ in the sandy unit and decreases in the shaly unit (Fig. 4). $\mathrm{K}_{2} \mathrm{O}$ ranges between $0.29-1.51 \%$ with an average values of $0.89 \%$. $\mathrm{CaO}$ ranges (Fig. 5) from 0.28 to $31.44 \%$. A maximum concentration was recorded at the depth of $2484 \mathrm{~m}(31.44 \%)$ and indicates presence of calcareous material and/or organic bands. K/Al ratio ranges from 0.07 to 0.50 and may indicate the presence of clay mineral such as illite [17]. Ti/Al ratios also display no clear trend; probably suggest aluminosilicate affiliation.

\subsection{Chemostratigraphic zonation}

Geochemical profiles provide a tool for recognizing stratigraphically significant geochemical events. These events are usually marked by either maxima or minima deflections in elemental curves [18,19]. However, based on interpretations made from the graphical analysis of the sediments geochemical data (Figs. 4-5) and chemostratigraphic correlation (Figs. 4-5), six chemostratigraphic zones (Table 3) have been established.

CHM 1 - This unit (Table 3) is characterized by high $\mathrm{CaO}$ and $\mathrm{Na}_{2} \mathrm{O}, \mathrm{Cr}, \mathrm{U}$ and $\mathrm{V}$. It corresponds to the base of the Imo Shale (Figs. 4 -5). CHM 2 - consists of shale and characterized by increase in concentration of $\mathrm{Ba}$ and $\mathrm{CaO}$. This may be due to the presence of fossil fragments. CHM 3 - corresponds to the upper section of the Imo shale, occurring between 1014 to $1284 \mathrm{~m}$; composed of sandstone, siltstone and high concentrations of Sr. CHM 4 - This chemofacies defines the upper part of the Ameki Formation, occurring between 684 to $1014 \mathrm{~m}$, made up of shaly sandstone, and high concentration of $\mathrm{K}_{2} \mathrm{O}$ and $\mathrm{Zr}$. CHM 5 - This unit correspond to Ameki Formation, made up of sandstone, and occur between 522 to $684 \mathrm{~m}$. High concentration of $\mathrm{CaO}$ and $\mathrm{Zn}$ (Table 3) dominates this zone. CHM 6 - This unit is characterized by the highest concentrations of $\mathrm{Fe}_{2} \mathrm{O}_{3}$, and $\mathrm{P}_{2} \mathrm{O}_{5}, \mathrm{Y}$ and Sc (Figs. 4 -5). 
Chemostratigraphy of Sediments in Benin West -1

Table 1. Trace element geochemistry of Benin West -1 .

\begin{tabular}{|c|c|c|c|c|c|c|c|c|c|c|c|c|c|c|c|c|c|c|c|c|c|c|}
\hline $\begin{array}{l}\text { Analyte symbol } \\
\text { Unit symbol } \\
\text { Detection Limit }\end{array}$ & $\begin{array}{l}\mathrm{Ag} \\
\mathrm{ppm} \\
0.3 \\
\end{array}$ & $\begin{array}{l}\mathrm{Al} \\
\% \\
0.01 \\
\end{array}$ & $\begin{array}{l}\text { As } \\
\text { ppm } \\
3 \\
\end{array}$ & $\begin{array}{l}\mathrm{Ba} \\
\mathrm{ppm} \\
7 \\
\end{array}$ & $\begin{array}{l}\mathrm{Be} \\
\mathrm{ppm} \\
1 \\
\end{array}$ & $\begin{array}{l}\mathrm{B} 1 \\
\mathrm{ppm} \\
2 \\
\end{array}$ & $\begin{array}{l}\mathrm{Ca} \\
\% \\
0.01 \\
\end{array}$ & $\begin{array}{l}\mathrm{Cd} \\
\mathrm{ppm} \\
0.3 \\
\end{array}$ & $\begin{array}{l}\text { Co } \\
\text { ppm } \\
1 \\
\end{array}$ & $\begin{array}{l}\mathrm{Cr} \\
\mathrm{ppm} \\
1 \\
\end{array}$ & $\begin{array}{l}\mathrm{Cu} \\
\mathrm{ppm} \\
1 \\
\end{array}$ & $\begin{array}{l}\mathrm{Fe} \\
\% \\
0.01 \\
\end{array}$ & $\begin{array}{l}\mathrm{Ga} \\
\mathrm{ppm} \\
1 \\
\end{array}$ & $\begin{array}{l}\mathrm{Hg} \\
\mathrm{ppm} \\
1 \\
\end{array}$ & $\begin{array}{l}\mathrm{K} \\
\% \\
0.01 \\
\end{array}$ & $\begin{array}{l}\mathrm{Li} \\
\mathrm{ppm} \\
1 \\
\end{array}$ & $\begin{array}{l}\mathrm{Mg} \\
\% \\
0.01 \\
\end{array}$ & $\begin{array}{l}\mathrm{Mn} \\
\mathrm{ppm} \\
1 \\
\end{array}$ & $\begin{array}{l}\text { Mo } \\
\text { ppm } \\
1 \\
\end{array}$ & $\begin{array}{l}\mathrm{Na} \\
\% \\
0.01 \\
\end{array}$ & $\begin{array}{l}\mathrm{Ni} \\
\mathrm{ppm} \\
1 \\
\end{array}$ & $\begin{array}{l}\mathrm{P} \\
\% \\
0.001 \\
\end{array}$ \\
\hline Analysis method & $\begin{array}{l}\text { TD- } \\
\text { ICP }\end{array}$ & $\begin{array}{l}\text { TD- } \\
\text { ICP }\end{array}$ & $\begin{array}{l}\text { TD- } \\
\text { ICP }\end{array}$ & $\begin{array}{l}\text { TD- } \\
\text { ICP }\end{array}$ & $\begin{array}{l}\text { TD- } \\
\text { ICP }\end{array}$ & $\begin{array}{l}\text { TD- } \\
\text { ICP }\end{array}$ & $\begin{array}{l}\text { TD- } \\
\text { ICP }\end{array}$ & $\begin{array}{l}\text { TD- } \\
\text { ICP }\end{array}$ & $\begin{array}{l}\text { TD- } \\
\text { ICP }\end{array}$ & $\begin{array}{l}\text { TD- } \\
\text { ICP }\end{array}$ & $\begin{array}{l}\text { TD- } \\
\text { ICP }\end{array}$ & $\begin{array}{l}\text { TD- } \\
\text { ICP }\end{array}$ & $\begin{array}{l}\text { TD- } \\
\text { ICP }\end{array}$ & $\begin{array}{l}\text { TD- } \\
\text { ICP }\end{array}$ & $\begin{array}{l}\text { TD- } \\
\text { ICP }\end{array}$ & $\begin{array}{l}\text { TD- } \\
\text { ICP }\end{array}$ & $\begin{array}{l}\text { TD- } \\
\text { ICP }\end{array}$ & $\begin{array}{l}\text { TD- } \\
\text { ICP }\end{array}$ & $\begin{array}{l}\text { TD- } \\
\text { ICP }\end{array}$ & $\begin{array}{l}\text { TD- } \\
\text { ICP }\end{array}$ & $\begin{array}{l}\text { TD- } \\
\text { ICP }\end{array}$ & $\begin{array}{l}\text { TD- } \\
\text { ICP }\end{array}$ \\
\hline $\begin{array}{l}\text { BENIN WEST-1 } \\
\text { WELL - } 234\end{array}$ & 0.7 & 2.57 & 20 & 198 & 2 & $<2$ & 0.32 & $<0.3$ & 14 & 45 & 10 & 1.92 & 12 & $<1$ & 0.36 & 28 & 0.13 & 177 & 2 & 0.11 & 19 & 0.016 \\
\hline $\begin{array}{l}\text { BENIN WEST-1 } \\
\text { WELL - } 522\end{array}$ & $<0.3$ & 4.36 & 25 & 691 & 5 & $<2$ & 1.59 & $<0.3$ & 19 & 65 & 11 & 6.40 & 19 & $<1$ & 0.88 & 40 & 0.41 & 1440 & 1 & 0.11 & 33 & 0.482 \\
\hline $\begin{array}{l}\text { BENIN WEST-1 } \\
\text { WELL - } 618\end{array}$ & 0.6 & 2.94 & 16 & 373 & 2 & $<2$ & 0.22 & 0.4 & 12 & 43 & 10 & 4.82 & 13 & $<1$ & 0.79 & 30 & 0.20 & 595 & 3 & 0.13 & 24 & 0.030 \\
\hline $\begin{array}{l}\text { BENIN WEST-1 } \\
\text { WELL - } 678\end{array}$ & 0.6 & 3.73 & 27 & 500 & 2 & $<2$ & 3.26 & $<0.3$ & 8 & 70 & 9 & 3.58 & 18 & $<1$ & 0.76 & 45 & 0.47 & 187 & 3 & 0.16 & 24 & 0.094 \\
\hline $\begin{array}{l}\text { BENIN WEST-1 } \\
\text { WELL - } 792\end{array}$ & 1.6 & 6.16 & 18 & 117 & 3 & 3 & 0.67 & $<0.3$ & 16 & 68 & 11 & 4.64 & 28 & $<1$ & 1.08 & 52 & 0.76 & 305 & 2 & 0.26 & 39 & 0.062 \\
\hline $\begin{array}{l}\text { BENIN WEST-1 } \\
\text { WELL - } 972\end{array}$ & 0.7 & 4.15 & 8 & 255 & 2 & $<2$ & 0.28 & $<0.3$ & 12 & 55 & 16 & 3.53 & 17 & $<1$ & 0.48 & 42 & 0.35 & 211 & 2 & 0.21 & 29 & 0.022 \\
\hline $\begin{array}{l}\text { BENIN WEST-1 } \\
\text { WELL - } 1014\end{array}$ & 4.8 & 2.48 & 20 & 307 & 2 & $<2$ & 2.88 & 0.3 & 9 & 58 & 15 & 2.47 & 10 & $<1$ & 0.48 & 133 & 2.95 & 351 & 3 & 0.35 & 55 & 0.303 \\
\hline $\begin{array}{l}\text { BENIN WEST-1 } \\
\text { WELL - } 1044\end{array}$ & 1.5 & 3.53 & 22 & 104 & 4 & $<2$ & 0.92 & 0.8 & 15 & 58 & 12 & 4.35 & 17 & $<1$ & 0.26 & 46 & 0.50 & 215 & 3 & 0.24 & 35 & 0.064 \\
\hline $\begin{array}{l}\text { BENIN WEST-1 } \\
\text { WELL - } 1284\end{array}$ & 1.6 & 4.87 & 11 & $\begin{array}{l}>10 \\
00\end{array}$ & 2 & $<2$ & 1.62 & 2.0 & 13 & 111 & 14 & 4.84 & 28 & $<1$ & 0.79 & 64 & 0.75 & 194 & 2 & 0.68 & 46 & 0.171 \\
\hline $\begin{array}{l}\text { BENIN WEST-1 } \\
\text { WELL - } 1620 \\
\text { BENIN WEST-1 } \\
\text { WELL - } 1854 \\
\text { BENIN WEST-1 } \\
\text { WELL - } 2148\end{array}$ & 3.5 & 4.54 & 6 & 85 & 2 & $<2$ & 3.30 & 3.2 & 13 & 174 & 14 & 4.06 & 25 & $<1$ & 0.67 & 61 & 1.83 & 272 & 3 & 0.62 & 45 & 0.197 \\
\hline $\begin{array}{l}\text { BENIN WEST-1 } \\
\text { WELL - } 2484\end{array}$ & 0.8 & 2.54 & 3 & 180 & 1 & $<2$ & 17.4 & 0.5 & 4 & 47 & 6 & 1.65 & 10 & $<1$ & 1.28 & 12 & 0.47 & 261 & 6 & 1.13 & 12 & 0.057 \\
\hline
\end{tabular}


Table 2. Trace and major elements geochemistry of Benin West -1 .

\begin{tabular}{|c|c|c|c|c|c|c|c|c|c|c|c|c|c|c|c|c|c|c|c|c|c|c|}
\hline Analyte symbol & $\mathrm{S}$ & Sc & $\mathrm{Sr}$ & $\mathrm{Te}$ & $\mathrm{Ti}$ & $\mathrm{Ti}$ & $\mathrm{U}$ & $\mathrm{V}$ & $\mathrm{W}$ & $\mathrm{Y}$ & $\mathrm{Zn}$ & $\mathrm{Zr}$ & $\mathrm{SiO}_{2}$ & $\mathrm{Al}_{2} \mathrm{O}_{3}$ & $\begin{array}{l}\mathrm{Fe}_{2} \mathrm{O}_{3} \\
(\mathrm{~T})\end{array}$ & $\mathrm{MnO}$ & $\mathrm{MgO}$ & $\mathrm{CaO}$ & $\mathrm{Na}_{2} \mathrm{O}$ & $\mathrm{K}_{2} \mathrm{O}$ & $\mathrm{TiO}_{2}$ & $\mathrm{P}_{2} \mathrm{O}_{5}$ \\
\hline Unit symbol & $\%$ & $\begin{array}{l}\mathrm{Pp} \\
\mathrm{m}\end{array}$ & ppm & ppm & $\%$ & $\begin{array}{l}\mathrm{pp} \\
\mathrm{m}\end{array}$ & ppm & $\mathrm{ppm}$ & ppm & ppm & $\mathrm{ppm}$ & $\mathrm{ppm}$ & $\%$ & $\%$ & $\%$ & $\%$ & $\%$ & $\%$ & $\%$ & $\%$ & $\%$ & $\%$ \\
\hline Detection Limit & 0.01 & 4 & 1 & 2 & 0.01 & 5 & 10 & 2 & 5 & 1 & 1 & 5 & 0.01 & 0.01 & 0.01 & 0.01 & 0.01 & 0.01 & 0.01 & 0.01 & 0.001 & 0.01 \\
\hline Analysis method & $\begin{array}{l}\text { TD- } \\
\text { ICP }\end{array}$ & $\begin{array}{l}\text { TD } \\
- \\
\text { ICP }\end{array}$ & $\begin{array}{l}\text { TD- } \\
\text { ICP }\end{array}$ & $\begin{array}{l}\text { TD- } \\
\text { ICP }\end{array}$ & $\begin{array}{l}\text { TD- } \\
\text { ICP }\end{array}$ & $\begin{array}{l}\text { TD- } \\
\text { ICP }\end{array}$ & $\begin{array}{l}\text { TD- } \\
\text { ICP }\end{array}$ & $\begin{array}{l}\text { TD- } \\
\text { ICP }\end{array}$ & $\begin{array}{l}\text { TD- } \\
\text { ICP }\end{array}$ & $\begin{array}{l}\text { TD- } \\
\text { ICP }\end{array}$ & $\begin{array}{l}\text { TD- } \\
\text { ICP }\end{array}$ & $\begin{array}{l}\text { TD- } \\
\text { ICP }\end{array}$ & $\begin{array}{l}\text { FUS- } \\
\text { ICP }\end{array}$ & $\begin{array}{l}\text { FUS- } \\
\text { ICP }\end{array}$ & $\begin{array}{l}\text { FUS- } \\
\text { ICP }\end{array}$ & $\begin{array}{l}\text { FUS- } \\
\text { ICP }\end{array}$ & $\begin{array}{l}\text { FUS- } \\
\text { ICP }\end{array}$ & $\begin{array}{l}\text { FUS- } \\
\text { ICP }\end{array}$ & $\begin{array}{l}\text { FUS- } \\
\text { ICP }\end{array}$ & $\begin{array}{l}\text { FUS- } \\
\text { ICP }\end{array}$ & $\begin{array}{l}\text { FUS- } \\
\text { ICP }\end{array}$ & $\begin{array}{l}\text { FUS- } \\
\text { ICP }\end{array}$ \\
\hline $\begin{array}{l}\text { BENIN WEST-1 } \\
\text { WELL - } 234\end{array}$ & 1.46 & 7 & 74 & 13 & 0.30 & $<5$ & $<10$ & 53 & $<5$ & 12 & 67 & 117 & 74.94 & 3.15 & 2.67 & 0.024 & 0.25 & 0.47 & 0.12 & 0.46 & 0.480 & 0.06 \\
\hline $\begin{array}{l}\text { BENIN WEST-1 } \\
\text { WELL - } 522\end{array}$ & 0.76 & 18 & 187 & 8 & 0.31 & $<5$ & $<10$ & 110 & $<5$ & 46 & 160 & 21 & 58.77 & 11.88 & 8.99 & 0.183 & 0.67 & 2.12 & 0.11 & 1.09 & 0.763 & 1.36 \\
\hline $\begin{array}{l}\text { BENIN WEST-1 } \\
\text { WELL - } 618\end{array}$ & 0.67 & 8 & 76 & 6 & 0.25 & $<5$ & $<10$ & 51 & $<5$ & 13 & 554 & 113 & 77.59 & 7.44 & 6.33 & 0.083 & 0.31 & 0.28 & 0.13 & 0.94 & 0.382 & 0.06 \\
\hline $\begin{array}{l}\text { BENIN WEST-1 } \\
\text { WELL - } 678\end{array}$ & 0.69 & 10 & 229 & 11 & 0.37 & $<5$ & $<10$ & 82 & 6 & 16 & 187 & 114 & 69.64 & 9.77 & 4.69 & 0.024 & 0.74 & 4.29 & 0.18 & 0.89 & 0.601 & 0.20 \\
\hline $\begin{array}{l}\text { BENIN WEST-1 } \\
\text { WELL - } 792\end{array}$ & 1.42 & 16 & 203 & 14 & 0.61 & $<5$ & $<10$ & 92 & 6 & 21 & 108 & 234 & 57.09 & 16.40 & 5.98 & 0.040 & 1.18 & 0.84 & 0.29 & 1.25 & 0.957 & 0.16 \\
\hline $\begin{array}{l}\text { BENIN WEST-1 } \\
\text { WELL - } 972\end{array}$ & 0.89 & 10 & 113 & 6 & 0.32 & $<5$ & $<10$ & 55 & $<5$ & 14 & 93 & 117 & 70.85 & 10.66 & 4.59 & 0.027 & 0.54 & 0.35 & 0.24 & 0.56 & 0.483 & 0.04 \\
\hline $\begin{array}{l}\text { BENIN WEST-1 } \\
\text { WELL - } 1014\end{array}$ & 0.61 & 9 & 465 & 4 & 0.24 & $<5$ & $<10$ & 60 & 7 & 18 & 179 & 20 & 56.51 & 6.53 & 3.39 & 0.046 & 4.93 & 4.04 & 0.43 & 0.62 & 0.408 & 0.73 \\
\hline $\begin{array}{l}\text { BENIN WEST-1 } \\
\text { WELL - } 1044\end{array}$ & 4.12 & 10 & 84 & 9 & 0.45 & $<5$ & $<10$ & 91 & $<5$ & 19 & 467 & 155 & 52.25 & 8.63 & 5.16 & 0.029 & 0.75 & 1.12 & 0.27 & 0.29 & 0.882 & 0.16 \\
\hline $\begin{array}{l}\text { BENIN WEST-1 } \\
\text { WELL - } 1284\end{array}$ & 2.00 & 13 & 235 & 14 & 0.60 & $<5$ & $<10$ & 114 & $<5$ & 18 & 122 & 196 & 47.35 & 16.75 & 7.00 & 0.025 & 1.48 & 2.64 & 0.89 & 1.05 & 1.042 & 0.41 \\
\hline $\begin{array}{l}\text { BENIN WEST-1 } \\
\text { WELL - } 1620\end{array}$ & 1.46 & 12 & 191 & 16 & 0.44 & $<5$ & $<10$ & 158 & $<5$ & 20 & 109 & 149 & 47.17 & 13.50 & 5.94 & 0.037 & 3.20 & 4.95 & 0.79 & 0.84 & 0.814 & 0.52 \\
\hline $\begin{array}{l}\text { BENIN WEST-1 } \\
\text { WELL - } 1854\end{array}$ & & & & & & & & & & & & & 47.34 & 14.67 & 6.27 & 0.042 & 3.82 & 2.21 & 0.91 & 0.95 & 0.797 & 0.18 \\
\hline $\begin{array}{l}\text { BENIN WEST-1 } \\
\text { WELL - } 2148\end{array}$ & & & & & & & & & & & & & 42.06 & 19.61 & 6.49 & 0.027 & 1.87 & 3.57 & 0.67 & 1.19 & 0.839 & 0.41 \\
\hline $\begin{array}{l}\text { BENIN WEST-1 } \\
\text { WELL - } 2484\end{array}$ & 0.70 & 6 & 391 & 4 & 0.19 & $<5$ & $<10$ & 34 & $<5$ & 13 & 27 & 77 & 27.22 & 6.82 & 2.30 & 0.038 & 0.82 & 31.44 & 1.37 & 1.51 & 0.345 & 0.16 \\
\hline
\end{tabular}




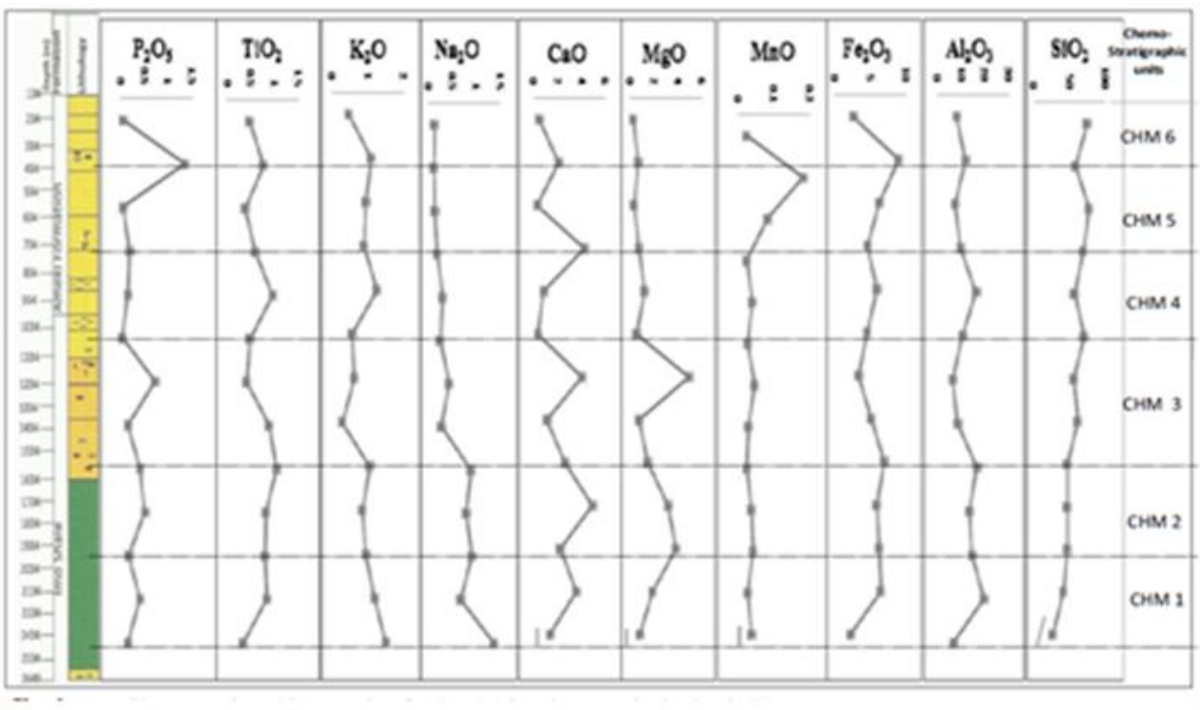

Fig.4. Chemostratigraphic zonation for the major elements in the Benin West-1.

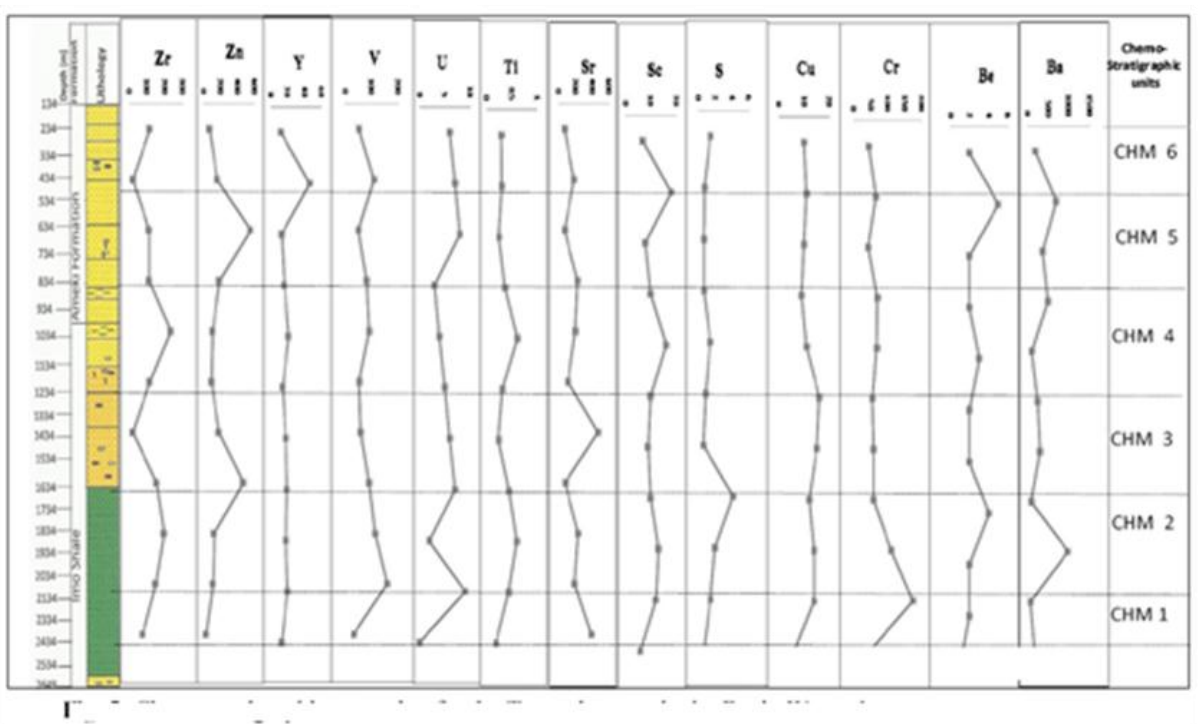

Fig. 5. Chemostratigraphic zonation for the trace elements in the Benin West-1. 
Table 3. Geochemical characteristics of chemostratigraphic zones of Benin west -1 well.

\begin{tabular}{|c|c|c|c|}
\hline Depth(m) & $\begin{array}{c}\text { Chemostratigraphic } \\
\text { Units }\end{array}$ & $\begin{array}{c}\text { Geochemical } \\
\text { Characteristics }\end{array}$ & Lithology \\
\hline 522 & CHM 6 & $\begin{array}{l}\quad 8.99 \% \mathrm{Fe}_{2} \mathrm{O}_{3}, \\
1.36 \% \mathrm{P}_{2} \mathrm{O}_{5}, \quad 46 \mathrm{ppm} \mathrm{Y}, \text { and } 18 \mathrm{ppm} \mathrm{Sc} .\end{array}$ & Sandstone \\
\hline 684 & CHM 5 & $4.29 \% \mathrm{CaO}, 187 \mathrm{ppm} \mathrm{Zn}$, & Sandstone \\
\hline 1014 & CHM 4 & $0.04 \% \mathrm{~K}_{2} \mathrm{O}, 234 \mathrm{ppm}, \mathrm{Zr}$, & Shaly sandstone \\
\hline 1284 & CHM 3 & $875 \mathrm{Zr}$ and $2745 \mathrm{ppm} \mathrm{Sr}$ & Sandstone \\
\hline 1854 & CHM 2 & $3.58 \% \mathrm{CaO}$ and $23630 \mathrm{ppm} \mathrm{Ba}$. & Shale \\
\hline 2484 & CHM 1 & $17.50 \% \mathrm{CaO}, 47 \mathrm{ppm} \mathrm{Cr}$, and $34 \mathrm{ppm} \mathrm{V}$ & Shale \\
\hline
\end{tabular}

\subsection{Paleoenvironmental implications}

Reconstruction of paleoenvironmental setting in the studied area is based on analysis of sedimentary attributes and chemofacies. Benin west -1 , contains diverse suites of lithologies; the upper section consists of fine - medium grained; poorly - moderately sorted sandstone which belong to Ameki Formation (134 - $934 \mathrm{~m})$. Grain size and coarsening/fining upward motifs indicate distributary/fluvial channel (Table 4) and barrier bar deposits of lower coastal plain settings [20,21]. The lower section (1034 $2649 \mathrm{~m}$ ) which belongs to Imo Shale, consists of both terrestrial organic matter, Monoporites annulatus, fossil shell fragments, foraminiferal wall linings and dinoflagellate cysts $[4,16]$. These sedimentary and palynological features suggest proximity to the paleo - shoreline [4,22]. Vanadium concentrations (Fig. 5) generally moderate in the sandy units $(51-110 \mathrm{ppm})$ and high in the shaly units (114 - 158 ppm). These indicate poorly oxygenated to euxinic condition [23,24]. Ba values generally vary between 11 and $300 \mathrm{ppm}$ [25]. Significantly, higher concentrations (up to $48350 \mathrm{ppm})$ occur in the middle and lower parts $(1014 \mathrm{~m})$ of the section (17055$48350 \mathrm{ppm})$ and suggest increased in bio-productivity [26,27]. 
Table 4. A Summary of sedimentological and geochemical fingerprints for the recognition of depositional environments in Benin West -1 .

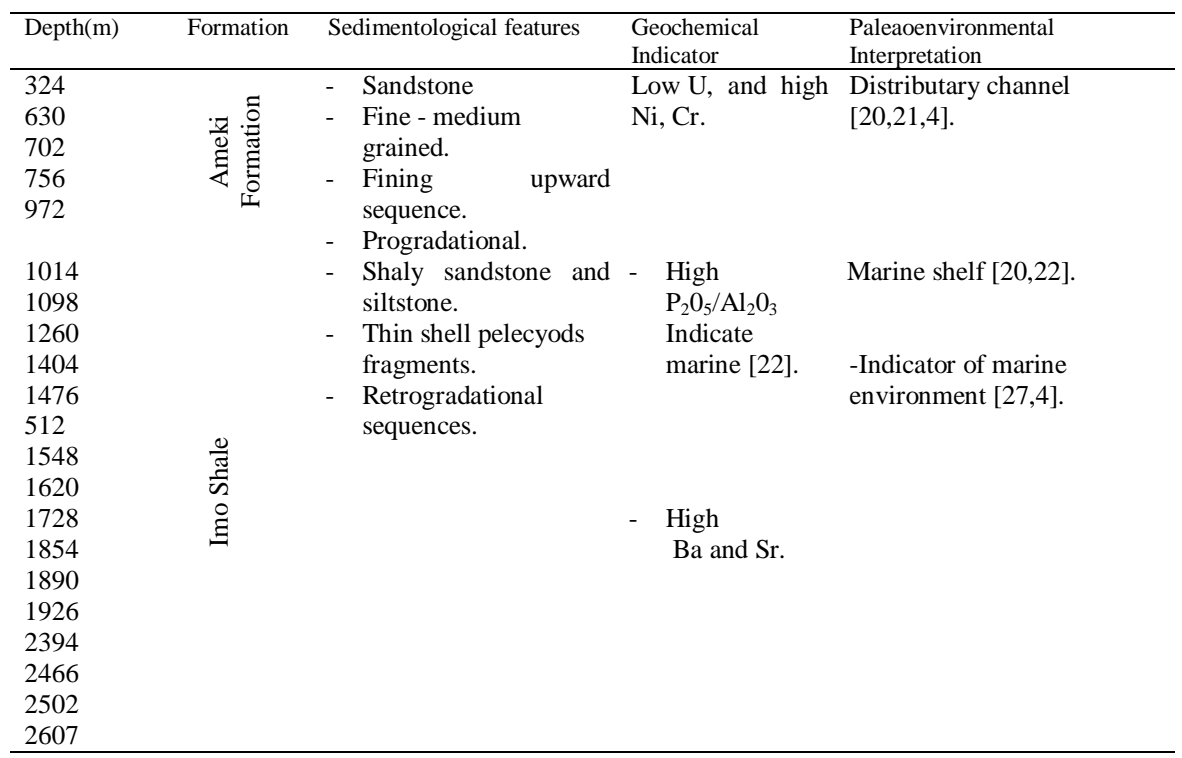

\section{Conclusion}

Integration of sedimentology and chemostratigraphic analyses of the sediments indicates the following findings:

i) Six chemozones (CHM 1, CHM 2, CHM 3, CHM 4, CHM 5 and CHM 6) are recognized in the sedimentary column.

ii) CHM 3 zone is delineated as a principal reservoir (quartz arenite) and capped with silty shales (probable effective seals).

iii) Sedimentological signatures and maxima peaks and /or fluctuating trends of $\mathrm{Zr}, \mathrm{SiO}_{2}, \mathrm{~K}_{2} \mathrm{O}, \mathrm{Sr}$ adjudged CHM 3 zone as a potential reservoir.

iv) Palaeoenvironment ranges from marine shelf -distributary channel.

\section{References}

1. G. Doughty, T. Pearce, and D. Mclean, Using Chemostratigraphy to Identify Reservoirs with Optimal Deliverability to Increase Gas Production from the Carboniferous (Pennsylvanian of the United Kingdom South North Sea. (Abstract), Am. Assoc. Petrol. Geol. (2006).

2. H. C. Jenkyns, Geochem. Geophys. Geosyst. 11(3), 1 (2010). http://dx.doi.org/10.1029/2009GC002788

3. K. T. Ratcliffe, A. Morton, D. Ritcey, and C. E. Evenchick, J. Canad. Petrol. Geol. 35, 320 (2007). http://dx.doi.org/10.2113/gscpgbull.55.4.320

4. O. Odedede, Ph.D Thesis, University of Benin, Benin City, Nigeria (2013).

5. K. T. Ratcliffe, A. M. Wright, and K. Schmidt, Soc. Sed. Geol. 10(12), 4 (2012). 
6. S. J. Coker and J. E. Ejedawe, J. Min. Geol. 23, 27 (1987).

7. E. I. Enu, Sed. Geol. 44, 65 (1985). http://dx.doi.org/10.1016/0037-0738(85)90032-6

8. J. Chêne, R. Onyike, and M. A. Sowumi, Rev. De Espan. Micro. Paleont. 10, 285 (1978).

9. J. Pierce and J. Parker, in Identifying Landing Zones Utilizing High- Resolution X-ray Fluorescence Chemostratigraphy - Unconventional Resources Technology Conference (Texas, USA, 2015). http://dx.doi.org/10.15530/urtec-20152154543

10. R. C. Murat, Stratigraphy and Paleogeography of Cretaceous and Lower Tertiary in Southern Nigeria: In African Geology, ed. T. F. J. Dessauvagie et al. (Ibadan University Press, Ibadan, Nigeria, 1972) pp. 251-256.

11. S. O. Nwachukwu, Geol. Mag. 109(50), 41 (1972). http://dx.doi.org/10.1017/S0016756800039790

12. P. M. P. Zaborski, Geosc. Rev. 5, 385 (1998).

13. M. Hoque, Sed. Geol. 17, 235 (1977). http://dx.doi.org/10.1016/0037-0738(77)90047-1

14. K. C. Short and J. Stauble, Am. Assoc. Petrol. Geol. Bull. 51, 761 (1967).

15. T. J. A. Reijers, S. W Petters, and C. S. Nwajide, Geology of the southern Anambra Basin: In Selected Chapters on Geology (SPDC corporate Reprographic Services, Warri, Nigeria, 1996).

16. F. A. Lucas and O. M. Odedede, World J. Eng. 9(6), 513 (2012). http://dx.doi.org/10.1260/1708-5284.9.6.513

17. M. Ortega-Huertas, P. Monaco, and L. Palomo, Clay Min. 28, 297 (1993). http://dx.doi.org/10.1180/claymin.1993.028.2.10

18. N. O. Jorgensen, Am. Assoc. Petrol. Geol. Bull. 70(3), 309 (1986)

19. I. M. Akaegbobi, U. O. Anyaeji, and G. O. Ogungbesan, Bul. Nig. Assoc. Petrol. Explor. 21(1), 53 (2009).

20. R. C. Selley, Ancient Sedimentary Environments, $3^{\text {rd }}$ edition (Chapman and Hall, London, 1984) pp. 305.

21. G. J. Nichols, Sedimentology and Stratigraphy (Blackwell Science, London, 1999) pp. 350.

22. T. J. Pearce, D. Mclean, J. H. Martin, K. Ratcliffe, and D. S. Wray, SEPM Spec. Publ. 94, 221 (2010).

23. M. D. Lewan and J. B. Maynard, Geochemica et Cosmochimica Acta 46, 2546 (1982). http://dx.doi.org/10.1016/0016-7037(82)90377-5

24. G. N. Breit and R. B. Wanty, Chem. Geol. 91, 83 (1991). http://dx.doi.org/10.1016/0009-2541(91)90083-4

25. A. Bellanca, A. Massetti, R. Neri, and F. Venezia, J. Sed. Res. 69(2), 466 (1999). http://dx.doi.org/10.2110/jsr.69.466

26. B. Schmitz, Palaeooceanography 2, 63 (1987). http://dx.doi.org/10.1029/PA002i001p00063

27. J. Dymond, E. Suess, and M. Lyle, Paleoceanography 7, 163 (1992). http://dx.doi.org/10.1029/92PA00181 